\title{
Influence of the ground on the sound screen efficiency
}

\author{
Suzanna Oganesyan and Alexandr Komkin* \\ B M STU, Power Engineering Department, 105005, M oscow, Russia
}

\begin{abstract}
Characteristic features of evaluating the acoustic efficiency of sound screen on the ground were considered. To simplify the analysis, noise source and control point were located symmetrically on both sides of the screen. Their position was determined by the angle $\alpha$ and by the effective screen height. Influence of the $\alpha$ on the efficiency of a semi-infinite screen, and then on a decrease in this efficiency in the presence of the ground was analyzed.
\end{abstract}

\section{Introduction}

Automobile traffic appears to be the main source of noise in urban environment. It is possible to reduce the effect of this noise in residential areas either by improving acoustic characteristics of primarily the vehicles, which is a very difficult task [1-3], or by installing noise-protective screens along the roads. Structurally, noise-protective screens differ in shape and materials used in their production. A fairly large number of publications both in our country and abroad were devoted to studying the noise-protective screens, both theoretically and experimentally [4-20]. Acoustic screen theoretical consideration is based on using the optical diffraction theory. However, this theory is very complicated and could be used only with a number of assumptions.

In practice, calculation of the acoustic screen efficiency is carried out using the Maekawa formula [4-6], which is the result of approximating the experimental data. Fresnel number is the basis of this formula, which, in addition to the sound wave length, also takes into account the difference in the path of sound rays in the acoustic screen presence and absence. This approach to calculation is attractable by its simplicity, but has certain limitations, since it is valid only for a semi-infinite absolutely rigid screen. Therefore, it is unable to take into account the ground surface, on which the screen is placed. Of course, characteristics both of the ground and of the screen material could not be taken into account in this situation. Taking these factors into consideration in calculating with analytical methods is rather difficult; therefore, very often the effect on this surface on the result obtained is neglected in technical acoustics. Though much attention is being paid to these issues [7-19], numerical calculations would remain the main tool in the near future [20]. This paper is using a simple analytical model to consider an screen located on the ground surface in order to study the influence of this surface on the screen efficiency.

* Corresponding author: akomkin@ mail.ru 


\section{Semi-infinite screen}

In the absence of the ground, the acoustic efficiency of the screen $\Delta L, \mathrm{~dB}$, will depend on the Fresnel number $N=2 \delta / \lambda$, where $\delta$ is the so-called path difference, showing how much the path travelled by the sound beam from the point source of sound to the calculated point through the top edge of the screen differs from the direct distance between the source and the calculation point; $\lambda$ is the sound wavelength. For $N \geq 1$, the $\Delta L$ value is determined by the Maekawa formula:

$$
\Delta L=10 \lg (20 N)
$$

Let us represent the formula (1) in the form of two components:

$$
\Delta L=10 \lg (40 \delta)+10 \lg (1 / \lambda)=\Delta L_{\delta}+\Delta L_{\lambda}
$$

The first of the components in (2) is determined only by the path difference, and the second - by the sound wave length. Since in this paper we are primarily interested in the influence of the mutual position of the noise source and the control point relative to the screen, in the future we will consider only the first component of the screen efficiency $\Delta L_{\delta}$.

The Maekawa formula determines the semi-infinite screen effectiveness and is surprisingly simple. It determines the problem geometry by only one parameter, i.e. the path difference. On the other hand, this parameter is not uniquely connected to specific location of the sound source and the control point relative to the screen. In order to make the screen acoustic efficiency analysis more tied to specific coordinates of the sound source and the control point, let us consider for simplicity that these points are located symmetrically relative to the screen.

At the first stage, let us consider such an approach as applied to a semi-infinite screen, which is shown in Figure 1.

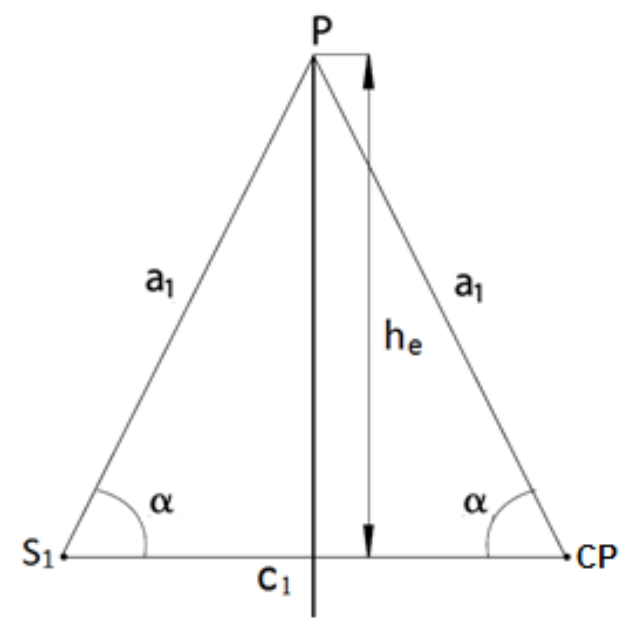

Fig. 1. Diagram of the semi-infinite screen.

In such a design diagram, the noise source and control point coordinates are set by the angle $\alpha$ and the effective screen height he. Path difference for such a model is: 


$$
\delta_{1}=2 a_{1}-c_{1}
$$

Given that:

$$
a_{1}=h_{e} / \sin \alpha ; c_{1}=2 h_{e} / \operatorname{tg} \alpha,
$$

the following is obtained:

$$
\delta_{1}=2 h_{e} \operatorname{tg}(\alpha / 2)
$$

$$
\begin{aligned}
& \text { At } \alpha \rightarrow 0 \operatorname{tg}(\alpha / 2) \rightarrow 0 \text { и } \delta_{1} \rightarrow 0 . \\
& \text { At } \alpha \rightarrow 90^{0} \operatorname{tg}(\alpha / 2) \rightarrow 1 \text { и } \delta_{1} \rightarrow 2 h_{e} .
\end{aligned}
$$

Thus, the following is obtained:

$$
\Delta L_{\delta 1}=10 \lg \left[80 h_{e} \operatorname{tg}(\alpha / 2)\right] .
$$

Figure 2 presents characteristics of a semi-infinite screen, which show that with an increase in the $\alpha$, the $\delta_{1}$ smoothly increases in accordance with the tangential dependence and at $\alpha \rightarrow 90^{\circ}$ reaches the $2 h_{e}$ value. As expected, the $\Delta L_{\delta 1}$ also increases with the growing $\alpha$ and $h_{e}$. As graphs in Figure 2a demonstrate, when the $h_{e}$ is doubled, the $\Delta L_{\delta 1}$ value increases by $3 \mathrm{~dB}$, which directly follows from formula (6). In addition, it follows from the graphs that when the $\alpha$ angle is altered from $15^{\circ}$ to $90^{\circ}$, the $\Delta L_{\delta 1}$ value increases by $9 \mathrm{~dB}$. In addition, in the considered region of variation $h_{e}$, the screen efficiency varies from $10 \mathrm{~dB}$ to $28 \mathrm{~dB}$.

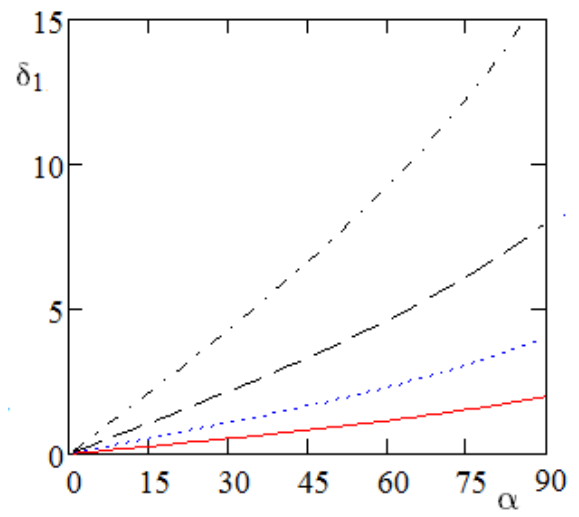

(a)

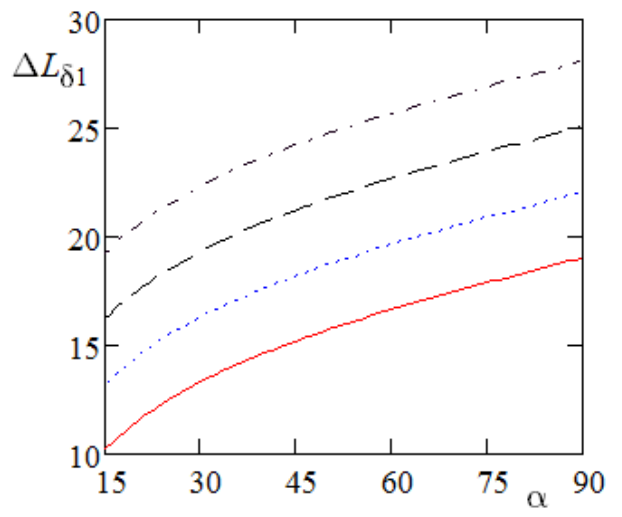

(b)

Fig. 2. Dependence of the path difference (a) and efficiency (b) for a semi-infinite screen on the system parameters: $(-)-h_{e}=1 \mathrm{~m} ;(\ldots .)-.h_{e}=2 \mathrm{~m} ;(---)-h_{e}=4 \mathrm{~m} ;(-\cdot-)-h_{e}=8 \mathrm{~m}$.

\section{Ground located in front or behind the screen}

Let us now consider the case, when the ground is positioned surface in front of the screen (Figure 3). It should be noted that another calculation parameter hg appears in this model and determines the height of the noise source position over the ground. Presence of the ground leads to the sound propagation second path directed to the to the calculation point; sound 
from the noise source first falls on the ground, then is reflected from it, and being diffracted at the screen upper edge finally reaches the calculation point. Formally interpreting this situation, it could be assumed that noise from two sources now comes to the calculation point. Source $S_{2}$ is added to source $S_{1}$.

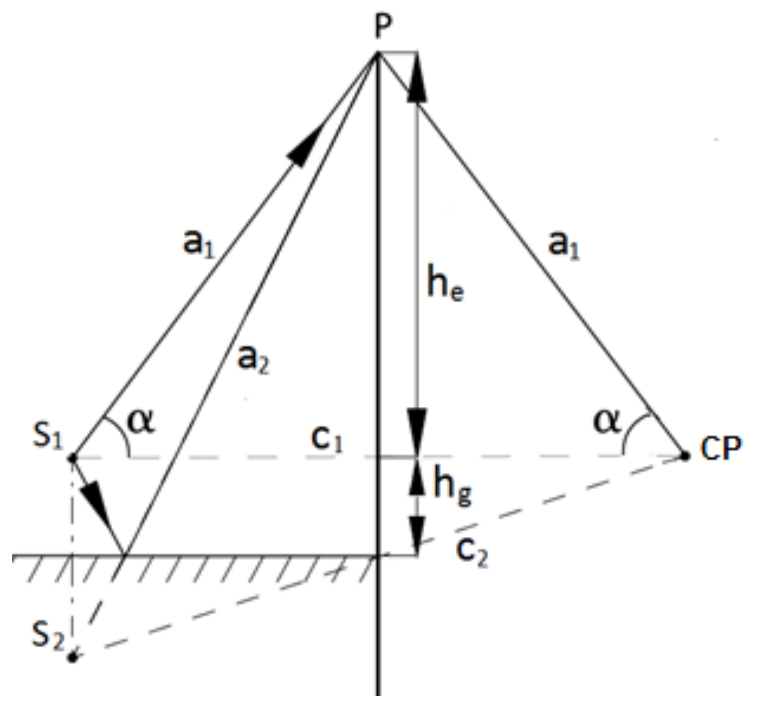

Fig. 3. Diagram with ground surface in front of the screen.

As follows from the diagram, path difference for the second source is:

$$
\delta_{2}=a_{2}-a_{1}-c_{2}
$$

where:

$$
\begin{aligned}
& a_{2}=\sqrt{\left(c_{1} / 2\right)^{2}+\left(h_{e}+2 h_{g}\right)^{2}} ; \\
& c_{2}=\sqrt{c_{1}^{2}+\left(2 h_{g}\right)^{2}} .
\end{aligned}
$$

In view of (4), expressions (8) and (9) are reduced to the following forms:

$$
\begin{aligned}
& a_{2}=h_{e} \sqrt{(1 / \operatorname{tg} \alpha)^{2}+\left(1+2 h_{g e}\right)^{2}} ; \\
& c_{2}=2 h_{e} \sqrt{(1 / \operatorname{tg} \alpha)^{2}+h_{g e}{ }^{2}},
\end{aligned}
$$

where $h_{g e}=h_{g} / h_{e}$.

Thus, screen efficiency for the second source is:

$$
\Delta L_{\delta 2}=10 \lg \left(40 \delta_{2} / R_{I}\right)
$$

where $R_{I}$ is the sound reflection coefficient from the ground surface (for the rigid surface $\left.R_{I .}=1\right)$. 
For comparison, Figure 4 presents characteristics of the path difference and the efficiency for a semi-infinite screen with $h_{e}=6 \mathrm{~m}$ and for a screen of the same effective height with a ground surface in front of it (source $S_{2}$ ). As could be seen (Figure 4a) path difference remains constant at the boundaries of the interval variation $\alpha\left[0 ; 90^{\circ}\right]$; however, the nature of the dependencies for different he changes within this interval. If for a semi-infinite screen this dependence is described by a concave curve, in the presence of a ground the corresponding curves become convex, and the higher is the hge parameter value, the more convex the curve becomes.

Further, as can be seen in Figure 4b, the value of screen efficiency for sources $S_{1}$ and $S_{2}$ remains unchanged at $\alpha=90^{\circ}$. However, at lower values of $\alpha$, the efficiency of sound screening of the source $S_{2}$ becomes higher than that of the source $S_{1}$, and the higher the value of the parameter hge, the greater this difference becomes.

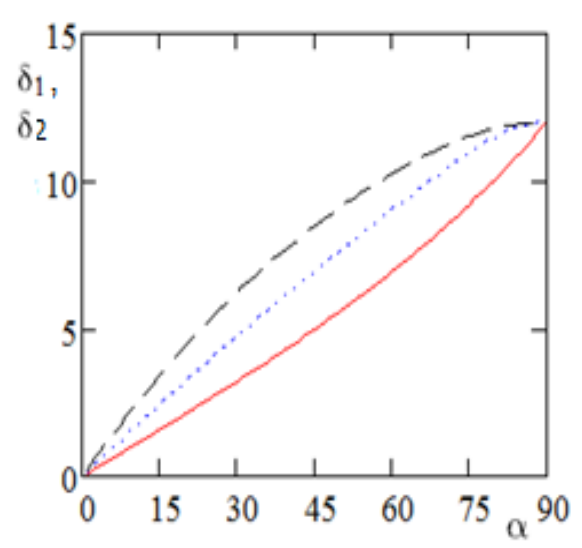

(a)

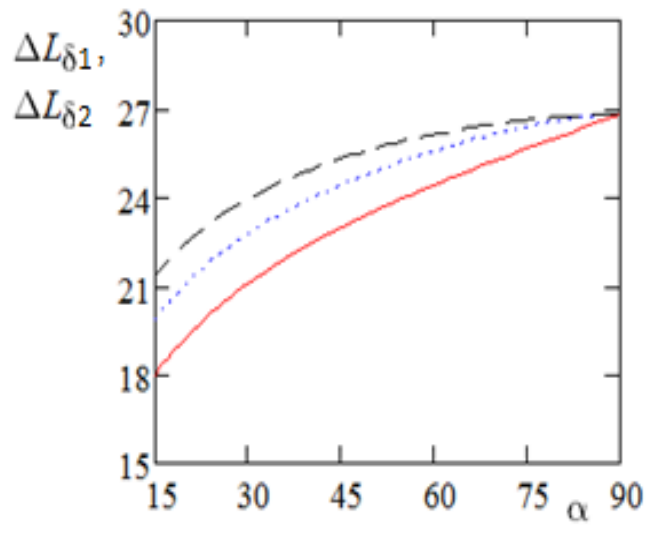

(b)

Fig. 4. Dependence of the path difference (a) and of the efficiency (b) on the system parameters for a semi-infinite screen with he $=6 \mathrm{~m}(-)$ and in the presence of ground surface in front of the screen with the same $h_{e}$ and $h_{g e}=1 / 2(\ldots .$.$) and h_{g e}=1 / 4(--)$.

Let us now determine the screen total efficiency for this model. Let us denote the sound presser level at the control point in the absence of a screen as $L_{p 0}$. Obviously, if a screen is installed, sound presser levels at the control point from sources $S_{1}$ and $S_{2}$ are determined respectively by the following expressions:

$$
L_{p 1}=L_{p 0}-\Delta L_{1} \text { and } L_{p 2}=L_{p 0}-\Delta L_{2}
$$

Let us assume in subsequent calculations that the primary $S_{1}$ and the secondary $S_{2}$ sources are uncorrelated. As in practice, the noise, as a rule, is of random and not of harmonic character, this assumption could be considered rightful. On this basis further on, it is possible to use the rule of logarithmic reduction in the sound pressure levels. In accordance with this, sound pressure level in the control point is determined by the following formula:

or

$$
L_{p \Sigma}=10 \log \left[10^{0,1 L_{p 1}}+10^{0,1 L_{p 2}}\right]=L_{p 1}+10 \log \left[1+10^{0,1\left(L_{p 1}-L_{p 2}\right)}\right]
$$

$$
L_{p \Sigma}=L_{p 0}-10 \lg (1 / \lambda)-\Delta L_{\delta 1}+10 \log \left[1+10^{0,1\left(\Delta L_{\delta 1}-\Delta L_{\delta 2}\right)}\right]
$$

Screen efficiency in (15) is determined by the last three terms. If we restricted to the terms associated with the path difference, the following is obtained: 


$$
\Delta L_{\delta \Sigma}=\Delta L_{\delta 1}-10 \log \left[1+10^{0,1\left(\Delta L_{\delta 1}-\Delta L_{\delta 2}\right)}\right]
$$

Since in (16) expression under the logarithm is always greater than 1, and that means the logarithm itself takes only positive values, then, as expected, a ground surface positioned in front of the screen leads to reducing its acoustic efficiency in comparison with the infinite screen efficiency.

Figure 5a presents for comparison graphical dependences of the total screen efficiency in the absence and presence of a ground surface in front of the screen on the system parameters. It follows from these graphs that taking into account reflection from the ground significantly reduces the total screen efficiency of the screen and this decrease could reach $3 \mathrm{~dB}$ at large values of $\alpha$. efficiency of the screen; therefor, this decrease could reach $3 \mathrm{~dB}$ at high $\alpha$.

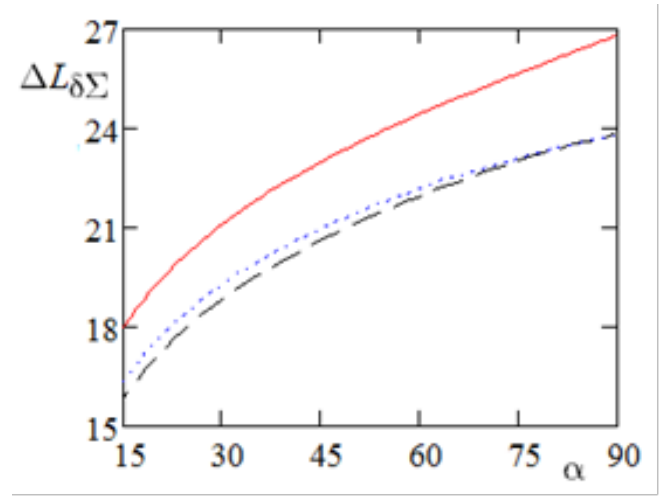

Fig. 5. Efficiency of a screen exposed to two sources at $h_{e}=6 \mathrm{~m}, h_{g e}=1 / 2(\ldots .$.$) and h_{g e}=1 / 4(--$-) with the semi-infinite screen efficiency $(-)$.

Let us now consider the case, when the ground surface is located behind a screen (Figure 6). Ground installed leads to the fact that there appears another sound propagation path to the control point, i.e. the sound from the source $S_{1}$ diffracts at the screen upper edge, falls on the ground, and then is reflected from it getting to the control point. Formally interpreting this situation, we can assume that noise from two sources now reaches the calculation point. Source $S_{3}$ is added to source $S_{1}$.

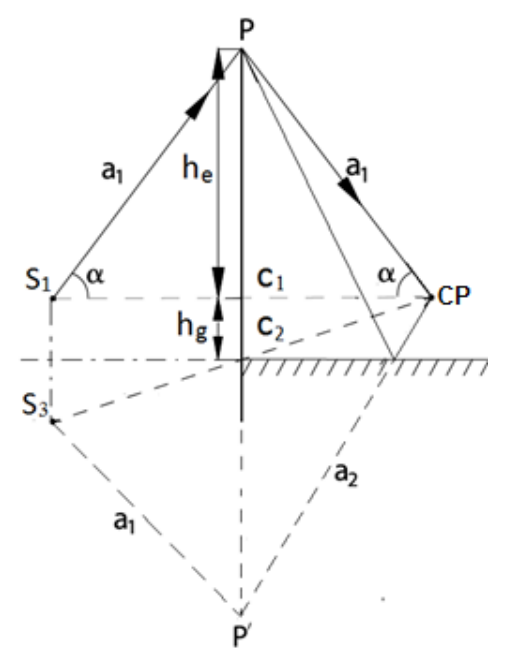

Fig. 6. Diagram with ground behind the screen. 
For the source $S_{3}$, the path difference is:

$$
\delta_{3}=a_{1}+a_{2}-c_{2}=\delta_{2}
$$

It follows that for the source $S_{3}$ screen efficiency is the same as for the source $S_{2}$ :

$$
\Delta L_{\delta 3}=\Delta L_{\delta 2}
$$

Considering the case, when the sound simultaneously from three sources $S_{1}, S_{2}$, and $S_{3}$ gets to the control point, and taking into account (18) we obtain by analogy with (16), the following formula for the total screen efficiency:

$$
\Delta L_{\delta \Sigma}=\Delta L_{\delta 1}-10 \log \left[1+2 \cdot 10^{0,1\left(\Delta L_{\delta 1}-\Delta L_{\delta 2}\right)}\right]
$$

In this case, the same graphical dependences could be received as in Figure 5, with the difference that the efficiency curve is located lower by about $1.5 \mathrm{~dB}$.

\section{Screen on the ground}

Let us now consider a practically important case, when the screen is positioned on the ground surface (Figure 7). In this case, the sound will reach the control point in one more way, when it falls on the ground from the source $S_{1}$, reflects from it and diffracts at the upper edge of the screen, after which it falls back onto the ground and, finally, again reflected from it, reaches the control point. Formally interpreting this situation, it could be assumed that such a path is provided by another source, i.e. $S_{4}$. Thus, there are already four sources of sound, one real $S_{1}$ and three virtual $\left(S_{2}, S_{3}, S_{4}\right)$ in the model under consideration.

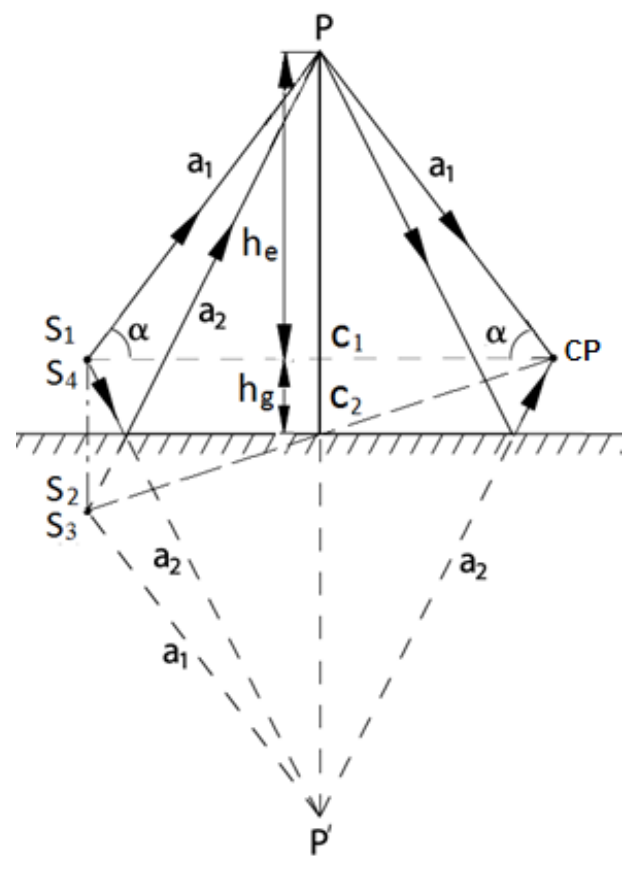

Fig. 7. Diagram of a screen on the ground. 
As follows from the diagram, the path difference for source $S_{4}$

$$
\delta_{4}=2 a_{2}-c_{1}
$$

Then, screen efficiency for the fourth source is:

$$
\Delta L_{\delta 4}=10 \lg \left(40 \delta_{4} / R_{I}^{2}\right) \text {. }
$$

Figure 8 presents for comparison characteristics of the path difference and of the acoustic efficiency of a semi-infinite screen (source $S_{1}$ ) and a screen on the ground for the source $S_{4}$.

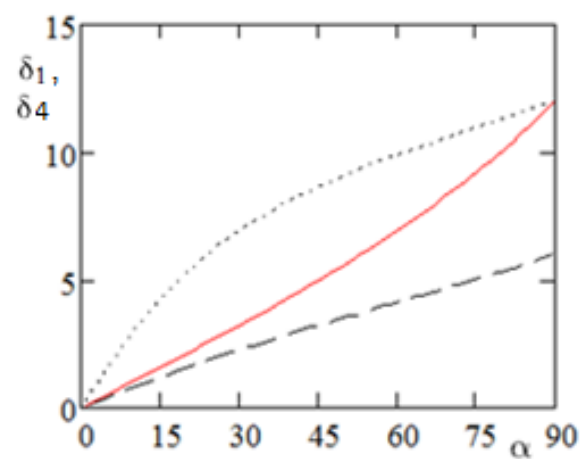

(a)

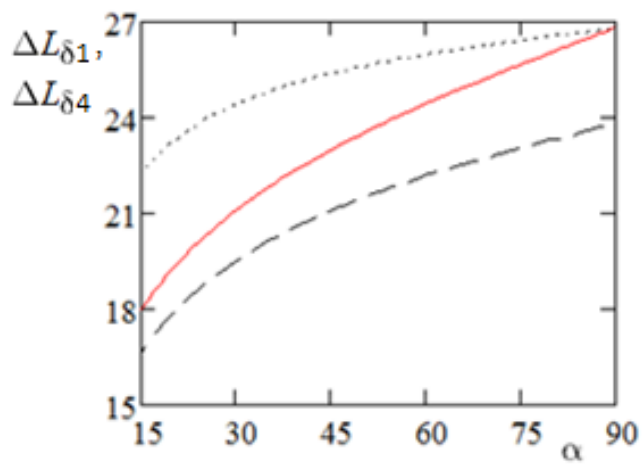

(b)

Fig. 8. Dependence of the path difference (a) and screen efficiency (b) on the system parameters for a semi-infinite screen with he $=6 \mathrm{~m}(-)$ and for a screen on the ground with $h_{e}=2 \mathrm{~m}$ and $h_{g e}=1(\ldots$. and $h_{g e}=1 / 4(--)$.

As follows from Figure 8a, path difference for source $S_{4}$ substantially depends on the hge and grows significantly with increase of $\alpha$. In general, path difference $\delta_{4}$ is much larger for the same effective screen height he than the path difference $\delta_{1}$. Since for the angle $\alpha=900$, the path differences are $\delta_{4}=2 h_{e}\left(1+2 h_{g e}\right)$ and $\delta_{1}=2 h_{e}$, so with $h_{g e}=1$, we get $\delta_{4}=3 \delta_{1}$.

Figure $8 \mathrm{~b}$ presents dependences of the screen efficiency for sources $\mathrm{S}_{1}$ and $\mathrm{S}_{4}$, which also show that, in general, suppression of noise from the fourth source is more significant than from the first source with the equal effective screen height of he. In addition, screen efficiency for source $S_{4}$ grows significantly with increasing values of the $h_{\text {ge }}$ parameter.

Let's now determine the overall efficiency of a screen placed on the ground. To do this, we need to consider the case, when sound from four noise sources $S_{1}, S_{2}, S_{3}$ and $S_{4}$ simultaneously arrive at the control point. Taking into account (21) and by analogy with (16) and (19), we obtain the following formula for the total screen efficiency:

$$
\Delta L_{\delta \Sigma}=\Delta L_{\delta 1}-10 \log \left[1+2 \cdot 10^{0,1\left(\Delta L_{\delta 1}-\Delta L_{\delta 2}+20 \lg R_{I}\right)}+10^{0,1\left(\Delta L_{\delta 1}-\Delta L_{\delta 4}+10 \lg R_{I}\right)}\right]
$$

Note that formula (22) is written taking into account the Ri reflection coefficient from the ground, while the above formulas and results were obtained for a rigid ground with $R_{I}=1$.

Figure 9 presents comparison of efficiencies of screen on the ground and of the semiinfinite screen with the $h_{e}=6 \mathrm{~m}$, which shows that presence of a ground reduces the screen efficiency compared to the semi-infinite screen, and this decrease at high $\alpha$ values and $h_{g e}=$ $1 / 4$ reaches almost $6 \mathrm{~dB}$. With an increase in the relative height hge, the screen efficiency becomes somewhat lower, especially at low values of $\alpha$. 


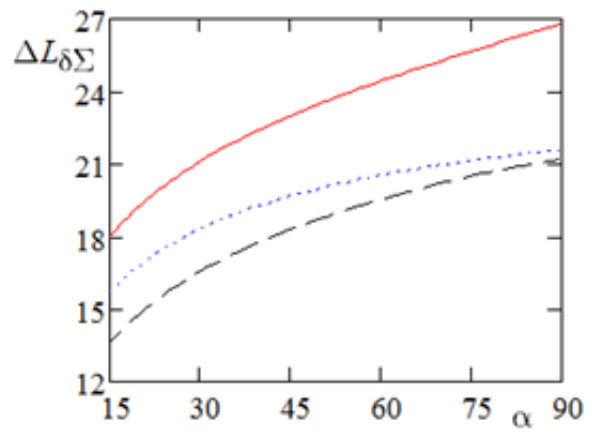

Fig. 9. Comparison of the screen efficiencies with $h_{e}=6 \mathrm{~m}$ located on the ground with $h_{g e}=1(\ldots .),. h_{g e}=1 / 4(--)$, $K=1$, and a semi-infinite screen $(-)$.

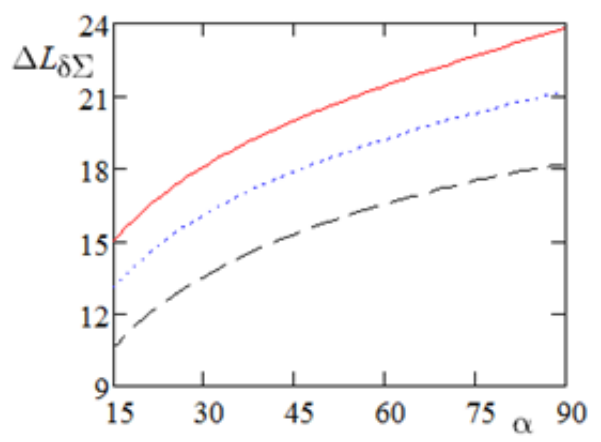

Fig. 10. Comparison of the screen efficiencies with $h_{e}=3 \mathrm{~m}$ located on the ground with $h_{g e}=1 / 4, K=0.5(\ldots .),. K=1$ $(--)$ and a semi-infinite screen $(-)$.

Figure 10 presents comparison of efficiencies of a screen on the ground and a semiinfinite screen with the same $h_{e}=3 \mathrm{~m}$, which shows that a decrease in the $R_{I}$ reflection coefficient of the ground, as expected, increases the efficiency of a screen on the ground. At the same time, comparison of the results in Figure 9 and Figure 10 demonstrates that, the decrease in screen efficiency due to presence of the ground does not depend on the effective screen height.

\section{Conclusion}

Studies conducted made it possible to draw the following conclusions.

1. To simplify analysis of the acoustic screen efficiency without losing its generality, it is advisable to consider symmetrical location of the sound source and of the control point relative to the screen. In this case, their position is determined by the effective height of the screen he and the angle a.

2. To assess dependence of the screen efficiency on the system parameters, it is advisable to introduce into consideration the efficiency component $\Delta L_{\delta}$ related to the path difference $\delta$, thus abstracting from the sound frequency.

3. Ground surface is able to significantly reduce the screen acoustic efficiency.

4. Magnitude of the screen efficiency decrease on a rigid ground could reach almost $6 \mathrm{~dB}$. This magnitude does not depend on the screen effective height, but it could be reduced by decreasing the ground reflection coefficient.

\section{References}

1. A. I. Komkin, A coust. Phys. 56 (3), 373 (2010)

2. N. D. Chaynov, V. A. Markov, A. A. Savastenko, Structural Noise and Acoustic Characteristics Improvement of Transport Power Plants, in IOP Conference Series: M aterials Science and Engineering, 2018, 327, 022069 (2018)

3. R. I. Rakhmatov, V. E. K rutolapov, V. N. Zuzov, Vibroengineering Procedia, 25, (6) 135 (2019) 
4. Z. M aekawa, A ppl. A coust. 1, 157 (1968)

5. Z. M aekawa, Acoustic barriers, in Noise reduction in buildings and residential areas, Ed. by F.L. Osipov and E.Y a. Y udin. (Stroyizdat, M oscow, 1987) [in R usssian]

6. Z. M aekawa, Recent Problems with Noise Barriers, in Proceedings of Noise-93 Conference, 31 M ay 31- 3 J une 1993, St. Petersburg, Russia (1993)

7. U. J. Kurze, J. A coust. Soc. A m. 55, 504 (1974)

8. T. Isei, T. F.W. Embleton, J. E. Piercy, J. A coust. Soc. A m. 67, 46 (1980)

9. A. L (i)sperance, J. Nicolas, G. A. Daigle, J. A coust. Soc. A m. 86, 1060 (1989)

10. S. I. Hayek, A ppl. A coust. 31, 77 (1990)

11. K. Takagi, A ppl. A coust. 31, 119 (1990)

12. Y. W. Lam, A ppl. A coust. 42, 29 (1994)

13. A. M uradaliand, K. R. Fyfe, A ppl. A coust. 53, 49 (1998)

14. P. M enounou, I. J. Busch-V ishniac, D. T. Blackstock, J. A coust. Soc. A m. 107, 2973 (2000)

15. P. M enounou, J. A coust. Soc. Am. 110, 1828 (2001)

16. P. M enounou, E. S. Papaefthymiou, A ppl. A coust. 71, 351 (2010)

17. A. I. Komkin, M .A. M ironov, S. I. Y udin, Simulation of acoustic radiation of the vehicle exhaust system above the ground, in Proceedings of Scientific Conference on the $100^{\text {th }}$ anniversary of A.V. Rimsky-K orsakov, M oscow, Russia. (2010) [in Russsian]

18. I. L. Shubin, I. E. Tsukernikov, N. Nikolov, A. Pisarsky. Basics of designing noise barriers (BASTET, M oscow, 2015) [in Russsian]

19. V. A. Kulkina, A. I. Komkin, Sound propagation over underlying surface, in in this Conference Proceedings

20. V. A . Kulkina, A . I. K omkin, A I. Bykov, Study of the acoustic characteristics of noise barriers, in this Conference Proceedings 